BULL. AUSTRAL. MATH. SOC.

VOL. 21 (1980), 373-391.

\title{
ON ONE-SIDED BOUNDEDNESS OF NORMED PARTIAL SUMS
}

\author{
R.A. MaLLer
}

\begin{abstract}
This paper gives a very general sufficient condition for the existence of constants $B(n), C(n)$ for which either $\underset{n \rightarrow+\infty}{\limsup } S_{n} / B(n)=1$ almost surely or $\underset{n \rightarrow+\infty}{\liminf } S_{n} / C(n)=-1$ almost surely, where $S_{n}=X_{1}+X_{2}+\ldots+X_{n}$ and $x_{i}$ are independent and identically distributed random variables. The theorem is closely connected with results of Klass and Teicher on the onesided boundedness of $S_{n}$, with the relative stability of $S_{n}$, and with a generalised law of the iterated logarithm due to Kesten. For non negative $X_{i}$ the sufficient condition is shown to be necessary, and the results are partially generalised to the case when $X_{i}$ form a stationary $m$-dependent sequence. Some connections with a generalised type of regular variation and with domains of partial attraction are also noted.
\end{abstract}

\section{Results}

Let $X_{i}, X$ be independent and identically distributed random variables with distribution $F$, and let $s_{n}=X_{1}+X_{2}+\ldots+X_{n}$. Suppose $P(|X| \geq x)>0$ for $x>0$. The first result of this paper is the following sufficient condition for one-sided boundedness of $S_{n}$, normed in an appropriate manner.

Received 19 November 1979. 
THEOREM 1. If

$$
\liminf _{x \rightarrow+\infty} x P(|X| \geq x) /\left|\int_{-x}^{x} u d F(u)\right|=0
$$

there are nondecreasing sequences $B(n), C(n)$, such that either $\underset{n \rightarrow+\infty}{\limsup } S_{n} / B(n)=1$ almost surely, or $\underset{n \rightarrow+\infty}{\liminf } S_{n} / C(n)=-1$ almost surely, or both.

This theorem is closely connected with the results of Klass [7], [8], and Klass and Teicher [9] on one-sided boundedness of $S_{n}$, and it is also connected with the relative stability of $S_{n} \cdot S_{n}$ is relatively stable if there is a sequence $b_{n} \rightarrow+\infty$ for which $s_{n} / b_{n} \stackrel{p}{\longrightarrow} 1$ or $s_{n} / b_{n} \stackrel{p}{\longrightarrow}-1$. It was shown by Rogozin [22] and Maller [13] that the relative stability of $s_{n}$ is equivalent to

$$
\lim _{x \rightarrow+\infty} x P(|X| \geq x) / \int_{-x}^{x} u d F(u)=0,
$$

which gives us the immediate corollary to Theorem 1 that, if $S_{n}$ is relatively stable, there are nondecreasing sequences $B(n), C(n)$, such that either $\underset{n \rightarrow+\infty}{\limsup } S_{n} / B(n)=1$ almost surely, or $\underset{n \rightarrow+\infty}{\liminf } S_{n} / C(n)=-1$ almost surely, or both. This also shows the connection of Theorem 1 with the results of Klass and Teicher, since in each of their theorems, $S_{n}$ is relatively stable. However, their extra restrictions, which include some kind of moment assumption, allow them to conclude something about the form of $B(n)$; in fact, that $B(n)$ may be computed from quantities involving only the distribution $F$ in a simple way. Due to the generality of Theorem 1, we cannot give any such representation for our norming sequence.

Theorem 1 is also closely connected with the result of Kesten [6], that if $F$ is in the domain of partial attraction of the normal distribution, equivalently

$$
\liminf _{x \rightarrow+\infty} x^{2} P(|X|>x) / \int_{-x}^{x} u^{2} d F(u)=0,
$$


there are sequences $\alpha_{n}, \gamma(n)$, for which

$$
-\infty<\liminf \left(s_{n}-\alpha_{n}\right) / \gamma(n)<\limsup \left(s_{n}-\alpha_{n}\right) / \gamma(n)<+\infty
$$

almost surely. This is a very general form of the law of iterated logarithm, and its converse is known to be true by results of Heyde [5] and Rogozin [20]. It was extended by Maller [16], who showed that $\gamma(n)$ may be chosen so that the almost sure limit points of $\left(s_{n}-\alpha_{n}\right) / \gamma(n)$ are precisely the interval $[-1,1]$. The methods of [6] and [16] are of great use in our proof of Theorem 1 . We note the similarity of the conditions

$$
\underset{x \rightarrow+\infty}{\liminf } x P(|X|>x) /\left|\int_{-x}^{x} u d F(u)\right|=0
$$

and

$$
\liminf _{x \rightarrow+\infty} x^{2} P(|x| \geq x) / \int_{-x}^{x} u^{2} d F(u)=0 ;
$$

This is a reflection of the duality between the convergence to normality of $S_{n}$ and its relative stability, as noticed by Raikov (see Gnedenko and Kolmogorov [3, p. 143] and Hall [4]).

We mention that the converse of Theorem 1 is not true, since of course any symmetric distribution in the domain of partial attraction of the normal distribution satisfies limsup $S_{n} / B(n)=1$ almost surely for some $B(n)$ by Kesten's result; but $\int_{-x}^{x} u d F(u) \equiv 0$ for such a distribution.

Even if we restrict attention to nonsymmetric distributions outside the domain of partial attraction of the normal distribution, there is no possibility of a converse to Theorem 1 , as is shown by the example on page 167 of Klass [7], together with his Theorem 1.2. (The fact that the distribution of Klass's random variable does not belong to the domain of partial attraction of the normal distribution follows from Theorem 1 of [15].)

Since the preparation of this paper a manuscript of a paper to appear by Pruitt [19] has been circulated. Theorem 5.2 of Pruitt's paper gives a result and a converse which includes our result of Theorem 1 . The methods 
and proofs of the two papers are quite different, and although Pruitt gives much more detail on the almost sure boundedness problem, he does not relate it to the concept of relative stability and its generalisations, nor does he consider any case other than independent and identically distributed random variables ( $c f$. our Theorems 2 and 3 ). Thus our approach to Theorem $I$ may be of independent interest.

For positive random variables, we can give a good summary.

THEOREM 2. Suppose $P(X \geq 0)=1$. Then the following are equivalent:

$$
\begin{aligned}
& \text { (i) } \underset{x \rightarrow+\infty}{\operatorname{Iiminf}} x P(X \geq x) / \int_{0}^{x} u d F(u)=0 \text {; } \\
& \text { (ii) } \underset{x \rightarrow+\infty}{\liminf } P(X \geq x \lambda) / P(X \geq x) \leq \lambda^{-1} \text { for } \lambda \geq 1 \text {; } \\
& \text { (iii) } \underset{x \rightarrow+\infty}{\liminf } \int_{0}^{x \lambda}[1-F(u)] d u / \int_{0}^{x}[1-F(u)] d u=1 \text { for } \lambda \geq 1 \text {; } \\
& \text { (iv) there are sequences } n_{i}++\infty, b_{n_{i}}++\infty \text {, for which } \\
& s_{n_{i}} / b_{n_{i}} \stackrel{p}{\longrightarrow} 1
\end{aligned}
$$

(v) there is a sequence $B(n) \rightarrow+\infty$ for which $\limsup _{n \rightarrow+\infty} S_{n} / B(n)=1$ almost surely.

Conditions (i), (ii), (iii), are purely analytical equivalences and are proved by some of the methods of the theory of regular variation. A curious consequence of ( $i i$ ) holding is that $F$ belongs to some domain of partial attraction ([15], Theorem 2). It is interesting to compare Theorem 2 with Theorem 2 of Rogozin [21], which pertains to the fluctuation properties of a relatively stable $S_{n}$. Then it will be seen that there are gaps in Theorem 2 concerned with properties of the characteristic function (or Laplace transform) which it would be useful to fill (in this context see also [14]). The equivalence of (i) and (iv) in Theorem 2 is proved in Theorem 3 of [13]. Note that (ii) holds if $P(X \geq x)$ is regularly varying with index less than or equal to -1 . 
As an application of Theorem 2 and Kesten's generalised law of the iterated logarithm it is easy to show that there are sequences $\alpha_{n}, \gamma(n)$, for which

$$
-\infty<\liminf _{n \rightarrow+\infty}\left(S_{n}-\alpha_{n}\right) / \gamma(n)<\underset{n \rightarrow+\infty}{\limsup }\left(S_{n}-\alpha_{n}\right) / \gamma(n)<+\infty
$$

almost surely if and only if there is a sequence $B(n)$ for which

$\underset{n \rightarrow+\infty}{\limsup } \sum_{i=1}^{n} x_{i}^{2} / B(n)=1$ almost surely, where the $x_{i}$ are not necessarily positive. This is an example of the "duality" principle of Raikov mentioned earlier.

Let $v(x)=\int_{0}^{x} u d F(u)$; in the case $X \geq 0$ almost surely, it is easy to deduce from $(i v)$ of Theorem 2 that $\liminf v(x \lambda) / v(x)=1$ for $\lambda \geq 1$. However, the converse to this is not true as may be shown by a minor modification of an example in [15], where an $F$ is given for which

$$
\liminf _{x \rightarrow+\infty} \int_{-x \lambda}^{x \lambda} u^{2} d F(u) / \int_{-x}^{x} u^{2} d F(u)=1 \text { for } \lambda \geq 1 \text {, }
$$

but $1-F(x)+F(-x)$ is slowly varying. (Simply take the same tail for a positive distribution.) It can be shown that

$$
\underset{x \rightarrow+\infty}{\liminf } \int_{0}^{x} u^{2} d F(u) / x \nu(x)=0
$$

if and only if

$$
\underset{x \rightarrow+\infty}{\liminf } v(x \lambda) / \nu(x)=1 \text { for } \lambda \geq 1 \text {, }
$$

so

$$
\liminf _{x \rightarrow+\infty} \int_{0}^{x} u^{2} d F(u) / x v(x)=0
$$

is necessary but not sufficient for (iv) of Theorem 2. (See Theorem 2 of [14].)

A paper of Miller [17] contains sufficient conditions for the 
existence of $C(n)$ with $\liminf _{n \rightarrow+\infty} S_{n} / C(n)=1$ almost surely. In fact, Miller's $C(n)$ may be taken as $n v(n)$ (he considers only the case $X \geq 0$ and $E X=+\infty)$. Miller's distributions satisfy $x[1-F(x)] / \int_{0}^{x} u d F(u) \rightarrow 0$, so they are relatively stable, and in particular ( $i$ ) of Theorem 2 holds. Thus they admit a sequence $B(n)$ for which limsup $S_{n} / B(n)=1$ almost surely, and this proves that $B(n)$ is not asymptotically equivalent to $n \nu(n)$; if it were, we would have $S_{n} / n v(n) \rightarrow 1$ almost surely, and, by the result of Chow and Robbins [1], EX<+o.

Consider the following proof, in essence due to Chow and Robbins [1], that ( $i v$ ) implies ( $v$ ) in Theorem 2: assume that the sequence in ( $i v$ ) increases rapidly enough for $S_{n_{i}} / b_{n_{i}} \rightarrow 1$ almost surely, and let $B(j)=b_{n_{i}}$ whenever $n_{i-1}<j \leq n_{i}$. Then $S_{n_{i}} / B\left(n_{i}\right) \rightarrow 1$ almost surely, so $\underset{n \rightarrow+\infty}{\limsup } S_{n} / B(n) \geq 1$ almost surely, while if $j>n_{1}$ and $i$ is such that

$$
n_{i-1}<j \leq n_{i}, \quad s_{j} / B(j) \leq \max _{n_{i-1}<j \leq n_{i}} S_{j} / B\left(n_{i}\right) \leq s_{n_{i}} / b_{n_{i}} \rightarrow 1
$$

almost surely, by the monotonicity of $S_{n}$ when $X \geq 0$ almost surely, showing that $\limsup _{n \rightarrow+\infty} S_{n} / B(n) \leq 1$ almost surely. Hence limsup $S_{n} / B(n)=1$ almost surely.

This argument does not require the independence (or even the identity of distributions) of the $x_{i}$, and it suggests that versions of Theorems 1 and 2 may be true under some form of dependence among the $X_{i}$. We say that $X_{i}$ forms a stationary sequence if the distribution of $\left(x_{1}, x_{2}, \ldots, x_{n}\right)$ is the same as that of $\left(x_{j+1}, x_{j+2}, \ldots, x_{j+n}\right)$ for $n \geq 1$ and $j \geq 1$, and that the $x_{j}$ are $m$-dependent if there is an integer $m \geq 0$ such that $X_{i}$ and $X_{j}$ are independent whenever $|i-j|>m$. With this definition, an independent sequence is 0 -dependent. We prove: 
THEOREM 3. Suppose $X_{i}$ is a stationary m-dependent sequence with marginal distribution $F$ for which

$$
x P(|X| \geq x) / \int_{-x}^{x} u d F(u)+0 \text { as } x \rightarrow+\infty .
$$

Then there are sequences $b_{n} \rightarrow+\infty, B(n) \rightarrow+\infty$, for which either

$S_{n} / b_{n} \stackrel{p}{\longrightarrow} 1$ and limsup $S_{n} / B(n)=1$ almost surely, or $S_{n} / b_{n} \stackrel{p}{\longrightarrow}-1$ and $\underset{n \rightarrow+\infty}{\liminf } S_{n} / B(n)=-1$ almost surely. The alternatives depend on the

ultimate sign of $\int_{-x}^{x} u d F(u)$, which is constant, and we have

$$
b_{n} \sim n\left|\int_{-b_{n}}^{b} u d F(u)\right|
$$

and $b_{n}$ is regularly varying with index 1 .

Theorem 3 partially generalises the results of [22], [7] and [8] to the m-dependent case.

\section{Proofs of theorems}

Proof of Theorem 1. We use the notations

$$
\begin{aligned}
& H(x)=P(|X| \geq x), \\
& \nu(x)=\int_{-x}^{x} u d F(u),
\end{aligned}
$$

and

$$
V(x)=\int_{-x}^{x} u^{2} d F(u)-\left[\int_{-x}^{x} u d F(u)\right]^{2},
$$

where $x>0$. When the condition of the theorem holds there is a sequence $x_{k} \uparrow+\infty$ for which $x_{k} H\left(x_{k}\right) / v\left(x_{k}\right) \rightarrow 0$, so $\left|v\left(x_{k}\right)\right|>0$ for large $k, X$ not being bounded above. By taking a subsequence, assume that $\nu\left(x_{k}\right)>0$; (this will lead to limsup $S_{n} / B(n)=1$, whereas assuming that $v\left(x_{k}\right)<0$ 
leads to liminf $\left.S_{n} / C(n)=-1\right)$.

We consider two cases. First, assume $x_{k}^{2} H\left(x_{k}\right) / V\left(x_{k}\right) \geq a>0$, and put $\zeta_{k}=x_{k} H\left(x_{k}\right) / \nu\left(x_{k}\right) \rightarrow 0$. By taking a subsequence if necessary, assume $\zeta_{k} \leq k^{-6}$. We have $v\left(x_{k}\right) / x_{k} \nu\left(x_{k}\right) \leq a^{-1} \zeta_{k}$. Define a sequence of integers $r_{k}$ as the integer part of $\log _{2}\left\{x_{k} \zeta_{k}^{-3 / 4} / \nu\left(x_{k}\right)\right\}$, where $\log _{2}$ stands for the logarithm to base 2 . Since $v\left(x_{k}\right) / x_{k} \rightarrow 0, r_{k} \rightarrow+\infty$. Let $x_{i}^{k}$ denote $x_{i}$ truncated at $\pm x_{k}$, and let $s_{n}^{k}=x_{1}^{k}+x_{2}^{k}+\ldots+x_{n}^{k}$. By Chebychev's inequality, if $y>0$,

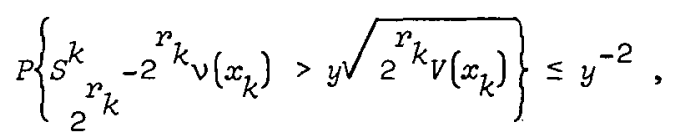

(1)

$$
P\left\{S_{{ }_{2}{ }_{r_{k}}}-2^{r_{k}}{ }_{\nu\left(x_{k}\right)}>-y \sqrt{2^{r_{k}}\left(x_{k}\right)}\right\} \geq 1-y^{-2} \text {. }
$$

We apply these inequalities with $y$ replaced by

$$
y_{k}=\delta 2^{r_{k}} v\left(x_{k}\right) / \sqrt{2^{r^{2}} k_{v}\left(x_{k}\right)}, \delta>0 .
$$

Note that

$$
\begin{aligned}
\delta^{-1} y_{k}=\left\{v^{2}\left(x_{k}\right) 2^{r_{k}} / v\left(x_{k}\right)\right\}^{\frac{3}{2}} & \sim\left\{\zeta_{k}^{-3 / 4} x_{k} v\left(x_{k}\right) / v\left(x_{k}\right)\right\}^{\frac{3}{2}} \geq a^{\frac{3}{2}} \zeta_{k}^{-7 / 8} \\
& \geq a^{\frac{3}{2} k^{21 / 4} \rightarrow+\infty},
\end{aligned}
$$

so an immediate consequence of (1) is that ${ }_{S_{2}^{k}}^{k}{ }_{r_{k}} / 2^{p_{k}} v\left(x_{k}\right) \stackrel{p}{\rightarrow} 1$. We have

$$
2^{r^{2} k_{H}}\left(x_{k}\right) \sim \zeta_{k}^{-3 / 4} x_{k} H\left(x_{k}\right) / \nu\left(x_{k}\right)=\zeta_{k}^{1 / 4} \leq k^{-3 / 2},
$$

which implies that the truncation may be disregarded to deduce that 
One-sided boundedness of normed partial sums

381

$S_{2}^{r_{k}}{ }^{r 2^{r}} v\left(x_{k}\right) \stackrel{\dot{p}}{\rightarrow} 1$. Define a sequence $B(n) \uparrow+\infty$ by

$$
B(n)=2^{r_{k}} v\left(x_{k}\right) \text { whenever } 2^{r k-1}<n \leq 2^{r_{k}} \text {; }
$$

then we have $S_{2}{ }_{r_{k}} / B\left(2^{r k}\right) \stackrel{p}{\longrightarrow} 1$, so limsup $S_{n} / B(n) \geq 1$ almost surely.

By a modification of Levy's inequality (Lemma 1 below), if $\delta>0$,

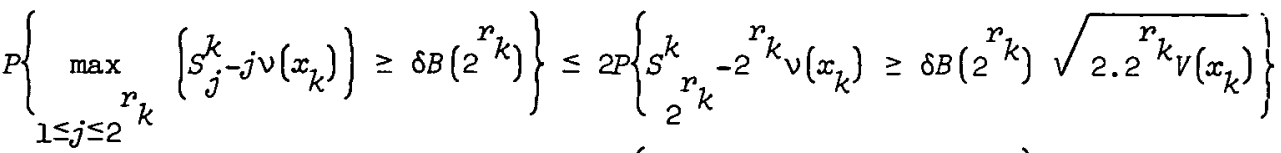

$$
\begin{aligned}
& \leq 2 P\left\{S^{k}{ }_{2}{ }_{r_{k}}-B\left(2^{r^{k}}\right) \geq \frac{3}{2} \delta B\left(2^{r}\right)\right\}
\end{aligned}
$$

if $k$ is large enough, since, as we showed above, $B^{2}\left(2^{r} k\right) / 2^{r} k_{V}\left(x_{k}\right) \rightarrow+\infty$. An easy consequence of ( 1 ) is that

$$
\sum P\left\{S_{2}^{k}{ }_{r_{k}}>(1+\delta) B\left(2^{r} k\right)\right\}
$$

converges when $\delta>0$, so we see that

$$
\sum P\left\{\max _{1 \leq j \leq 2} r_{k}\left(s_{j}^{k}-j v\left(x_{k}\right)\right) \geq \delta B\left(2^{r_{k}}\right)\right\}
$$

converges when $\delta>0$.

By the Borel-Cantelli Lemma, this means

$$
\limsup _{k \rightarrow+\infty} \max _{1 \leq j \leq 2} r_{k}\left(S_{j}^{k}-j \vee\left(x_{k}\right)\right) / B\left(2^{r}\right)=0
$$

almost surely, and so

https://doi.org/10.1017/S0004972700006237 Published online by Cambridge University Press 


$$
\begin{aligned}
\max _{1 \leq j \leq 2}{ }_{r_{k}} s_{j}^{k} / B\left(2^{r^{k}}\right) & \leq \max _{1 \leq j \leq 2} r_{k}\left(s_{j}^{k}-j \nu\left(x_{k}\right)\right) / B\left(2^{r^{k}}\right)+\max _{1 \leq j \leq 2}{ }^{r_{k}} j \nu\left(x_{k}\right) / B\left(2^{r_{k}}\right) \\
& o(1)+1 \text { almost surely as } k \rightarrow+\infty .
\end{aligned}
$$

Write $s_{j}=s_{j}^{k}+Y_{j}^{k}$, and note that

$$
\begin{aligned}
\sum_{k} P\left\{\max _{1 \leq j \leq 2}\left|Y_{j}^{k}\right|>0\right\} & =\sum_{k} P\left\{\left|Y_{j}^{k}\right|>0 \text { for some } j \leq 2^{r^{k}}\right\} \\
& \leq \sum_{k} P\left\{\left|x_{i}\right|>x_{k} \text { for some } i \leq 2^{r} k\right\} \\
& \leq \sum_{k} 2^{r} k_{H}\left(x_{k}\right)<+\infty
\end{aligned}
$$

so $\max _{1 \leq j \leq 2}\left|y_{j}^{k}\right| \rightarrow 0$ almost surely as $k \rightarrow+\infty$. This means

$$
\begin{aligned}
& \max _{1 \leq j \leq 2}{ }_{r_{k}} S_{j} / B\left(2^{r^{k}}\right) \leq \max _{1 \leq j \leq 2}{ }_{r_{k}} S_{j}^{k} / B\left(2^{r k}\right)+\max _{1 \leq j \leq 2} r_{k} r_{j}^{k} / B\left(2^{r k}\right) \\
& \leq 1+O(1) \text { almost surely. }
\end{aligned}
$$

If $n \geq 1$ choose $k=k(n)$ so that $2^{r_{k-1}}<n \leq 2^{r_{k}}$; then $B(n)=B\left(2^{r} k\right)$, and

$$
\underset{n}{\limsup } S_{n} / B(n) \leq \underset{n}{\limsup } \sup _{2^{r_{k-1}-j \leq 2} r_{k}^{r_{k}}} S_{j} / B\left(2^{r k}\right) \leq 1 \text { almost surely, }
$$

and we conclude that limsup $S_{n} / B(n)=1$ almost surely.

We come now to the second case, when $x_{k}^{2} H\left(x_{k}\right) / V\left(x_{k}\right) \rightarrow 0 . F$ is then in the domain of partial attraction of the normal distribution, and the methods we use are a modification of those in [16], which in turn is based on Kasten's wurk [6]. We put $\zeta_{k}=x_{k}^{2} H\left(x_{k}\right) / V\left(x_{k}\right)$, and assume that 
$\zeta_{k} \leq k^{-8}$. Let $r_{k}$ be the integer part of $\log _{2}\left\{\zeta_{k}^{-3 / 4} x_{k}^{2} / V\left(x_{k}\right)\right\}$, and again $x_{k} \rightarrow+\infty$. By the Berry-Esseen theorem (Feller [2, p. 542]), if、 $r \geq 1$,

$$
\sup _{-\infty<x<+\infty}\left|P\left\{S_{2^{k}}^{k}-2 \nu\left(x_{k}\right)<x \sqrt{2^{r} V\left(x_{k}\right)}\right\}-\Phi(x)\right| \leq L_{r}^{k}
$$

where

$$
\Phi(x)=(2 \pi)^{-\frac{1}{2}} \int_{-\infty}^{x} e^{-\frac{1}{2} u^{2}} d u \text { and } L_{r}^{k}=E\left|x_{i}^{k}-\nu\left(x_{k}\right)\right|^{3} / 2^{\frac{1}{2} r_{V} / 2}\left(x_{k}\right) .
$$

Exactly as in [16] we can show that

$$
\sum_{k \geq 1} \sum_{r_{k}-2 \log _{2} k<r \leq r_{k}} L_{r}^{k} \text { and } \sum_{k \geq 1} \sum_{r_{k-1}<r \leq r_{k}} 2^{r} H\left(x_{k}\right)
$$

are finite. Suppose firstly that

$$
y_{k}=2^{r_{k}}{ }\left(x_{k}\right) / \sqrt{2^{r} k_{V\left(x_{k}\right)}}
$$

contains a subsequence $\rightarrow+\infty$. By taking a subsequence throughout the proof we can then assume that $y_{k} / \log k \rightarrow+\infty$. Since $\sum L_{r_{k}}^{k}$ and $\sum 2^{r} k_{H}\left(x_{k}\right)$ are finite, we are in the situation of Case 1, with the inequality (2), with $r=r_{k}$, playing the same role as inequalities (1). We thus find that limsup $S_{n} / B(n)=1$ for the same $B(n)$ as before.

Alternatively we have $y_{k}$ bounded, in which case (2) implies

$$
\sum_{k \geq 1} \sum_{r_{k}-2 \log _{2} k<r \leq r_{k}} \sup _{-\infty<x<+\infty}\left|P\left\{X_{2}^{k}{ }_{r_{k}} \sqrt{2^{r^{2}} V\left(x_{k}\right)}<x+O(1)\right\}-\Phi(x)\right|<+\infty
$$

because, when $r \leq r_{k}$,

$$
2^{r} v\left(x_{k}\right) \sqrt{2^{r} V\left(x_{k}\right)}=2^{\frac{2}{2}\left(r-r_{k}\right)} 2^{r_{k}} v\left(x_{k}\right) \sqrt{2^{r} k_{V}\left(x_{k}\right)}=O(1) .
$$

Thus, putting $x=a \sqrt{2 \log \bar{k}}$, we see that 


$$
\sum_{k \geq 1} \sum_{r_{k}-2 \log 2^{k<r \leq r_{k}}} P\left\{S_{2^{k}}^{k}>a B\left(2^{r}\right)\right\}
$$

is finite when $a>1$, where we now define $B(n) \uparrow+\infty$ by

$$
B^{2}(n)=2.2^{r_{k}} V\left(x_{k}\right) \log k \text { when } 2^{r_{k-1}}<n \leq 2^{r^{r}} .
$$

By Chebychev's inequality

$$
\begin{aligned}
\sum_{k \geq 1} \sum_{r_{k-1}<r \leq r_{k}-2 \log _{2} k} P\left\{S_{2^{k}}^{k}-2^{r} \nu\left(x_{k}\right)>a B\left(2^{r}\right)\right\} & \\
& \leq a^{-2} \sum_{k \geq 1} \sum_{k-1} \sum_{r \leq r_{k}-2 l \log _{2} k} B^{-2}\left(2^{r}\right) 2^{r} V\left(x_{k}\right)<+\infty
\end{aligned}
$$

as in [16], and hence

$$
\sum_{k \geq 1} \sum_{r_{k-1}<r \leq r_{k}-2 \log _{2} k} P\left\{S_{2^{k}}^{k}>a B\left(2^{r}\right)\right\}<+\infty \text { when } a>1 \text {, }
$$

because when $r_{k-1}<r \leq r_{k}$,

$$
2^{r} v\left(x_{k}\right) / B\left(2^{r}\right)=2^{r} v\left(x_{k}\right) / B\left(2^{r}\right) \leq 2^{r} k_{\nu}\left(x_{k}\right) / \sqrt{2.2^{r} k_{v}\left(x_{k}\right) \log k}+0 .
$$

Thus we have

$$
\sum_{k \geq 1} \sum_{r_{k-1}<r \leq r_{k}} P\left\{S_{S^{k}}^{k}>a B\left(2^{r}\right)\right\}<+\infty \text { if } a>1 .
$$

The same is also true with $X_{i}$ replaced by $-X_{i}$, as a symmetric argument shows, and thus by symmetrising and ignoring the truncation, $\sum_{r \geq 1} P\left\{\left|S^{s}{ }_{2}^{p}\right|>2 a B\left(2^{r}\right)\right\}<+\infty$. By Lerma 2 of [6], this means limsup $S_{n}^{s} / B(n) \leq 4$ almost surely, so by Theorem 7 of $[6], S_{n}^{s} / B(n) \stackrel{p}{\longrightarrow} 0$, and hence, as in Feller $\left[2\right.$, p. 232], $\left(S_{n}-n \cup\left(B_{n}\right)\right) / B(n) \stackrel{p}{\longrightarrow} 0$.

If $n$ is large choose $k=k(n)$ so that $2^{r_{k-1}}<n \leq 2^{r_{k}}$; then 


$$
\begin{aligned}
& n B_{n}^{-1}|\nu(B(n))| \leq 2^{r_{k}}\left|\nu\left(B\left(2^{r^{k}}\right)\right)\right| / B\left(2^{r}\right) \leq 2^{r} k v\left(x_{k}\right) / B\left(2^{r} k\right) \\
& +2^{r^{2} k}\left|\int_{x_{k} \leq|u| \leq B\left(2^{r_{k}}\right)} u d F(u)\right| / B\left(2^{r} k\right) \\
& \leq o(1)+2^{r^{2}} k_{P}\left(|X|>x_{k}\right)=o(1) \text { as } n \rightarrow+\infty \text {, }
\end{aligned}
$$

so we deduce that $S_{n} / B(n) \stackrel{P}{\longrightarrow} 0$. We can now follow exactly the proof of [16] to obtain limsup $S_{n} / B(n)=1$ almost surely, and in fact that the limit points of $S_{n} / B(n)$ are precisely the interval $[-1,1]$. This concludes the proof of Theorem 1 .

Proof of Theorem 2. The equivalence of $(i)$ and (ii) is a simple consequence of Theorem 2 of [12], while the equivalence of ( $i$ ) and (iv) was shown in Theorem 3 of [13]. It is easy to see that (iv) implies (iii), while if $(i)$ does not hold,

$$
x[1-F(x)] / \int_{0}^{x}[1-F(u)] d u \geq \delta>0
$$

for $x$ large, so for such $x$ and $\lambda>1$,

$$
\begin{aligned}
\int_{0}^{x \lambda}[1-F(u)] d u / \int_{0}^{x}[1-F(u)] d u & =\exp \int_{x}^{x \lambda}[1-F(u)] d u / \int_{0}^{u}[1-F(y)] d y \\
& \geq \exp \delta\left(\int_{x}^{x \lambda} u^{-1} d u\right)=\lambda^{\delta},
\end{aligned}
$$

so (iii) does not hold. The fact that $(i)$ implies (v) follows from Theorem $l$, so it remains to show only that (v) implies ( $i)$. Suppose (v) holds and (i) does not, so (ii) also fails to hold. From Theorem 1 of [15] we then see that $F$ is not in the domain of partial attraction of the normal distribution, equivalently, by Lévy [10, p. 113], $x^{2} P(X \geq x) / V(x) \geq a>0$, where now

$$
V(x)=\int_{0}^{x} u^{2} d F(u)-\left[\int_{0}^{x} u d F(u)\right]^{2} .
$$

Since $S_{n} \geq X_{n}$, we have limsup $X_{n} / B(n) \leq 1$ almost surely, so by the 
Borel-Cantelli Lemma, $\sum P(X>2 B(n))$ converges, and hence $n P(X>2 B(n)) \rightarrow 0$. If limsup $n B^{-1}(n) \cup(2 B(n))>0$, where $v(x)=\int_{0}^{x} u d F(u)$, then $(i)$ holds, so assume $n B^{-1}(n) v(2 B(n)) \rightarrow 0$. This means $n^{-1} B(n) \rightarrow+\infty$. Let $X_{i}^{i}$ denote $X_{i}$ truncated at $2 B(i)$, and let $s_{n}^{n}=x_{1}^{1}+x_{2}^{2}+\ldots+x_{n}^{n}$. Since $\sum P(X>2 B(n))<+\infty, \quad\left(S_{n}-S_{n}^{n}\right) / B(n) \rightarrow 0$ almost surely. We also have

$$
\sum B^{-2}(n) \text { Var } X_{n}^{n}=\sum B^{-2}(n) V(2 B(n)) \leq 4 a^{-1} \sum P(X>2 B(n))<+\infty
$$

so by, for example, Loève [11, p. 252], $\left(S_{n}^{n}-E S_{n}^{n}\right) / B(n) \rightarrow 0$ almost surely. But

$$
E S_{n}^{n}=\sum_{i=1}^{n} E X_{i}^{i}=\sum_{i=1}^{n} \int_{0}^{2 B(i)} u d F(u) \leq n \nu(2 B(n))=o(B(n))
$$

so $S_{n}^{n} / B(n) \rightarrow 0$ almost surely and $S_{n} / B(n) \rightarrow 0$ almost surely. This contradiction completes the proof of Theorem 2.

Proof of Theorem 3. Let $A(x)=\int_{0}^{x}[1-F(u)-F(-u)] d u$. Since $x P(|X|>x) / \nu(x) \rightarrow 0, x P(|X|>x) / A(x) \rightarrow 0$, and since $A$ is continuous, $A$ is either positive or negative for large $x$, as in [13]. Suppose the former; then, as in [13], $A$ is slowly varying. Define a nondecreasing sequence $b_{n} \rightarrow+\infty$ by $b_{n}=\sup \left\{x>0: x^{-1} A(x) \geq n^{-1}\right\}$.

As in [21], we can show that $n P\left(|X|>x b_{n}\right) \rightarrow 0$ and $n b_{n}^{-2} V\left(x b_{n}\right) \rightarrow 0$ for $x>0$, that the sum of $n$ independent copies of $X$ is relatively stable, and also from [21], that $b_{n}$ is regularly varying with index 1 . Let $n_{m}$ be the integer part of $n /(m+1)$, and let

$$
s_{n_{m}}(t)=\sum_{i=1}^{n_{m}-1} x_{(m+1) i+t} \text {, for } 0 \leq t \leq m \text {. }
$$


Now $S_{n_{m}}(t)$ is the sum of $n_{m}-1$ independent and identically distributed random variables and hence is relatively stable with norming sequence $b_{n_{m}-1} ;$ clearly then, $S_{n_{m}}(t) / b_{n_{m}} \stackrel{p}{\longrightarrow} 1$. Since $b_{n}$ is regularly varying with index $1, S_{n_{m}}(t) / b_{n} \stackrel{p}{\rightarrow} 1 /(m+1)$, which means

$$
s_{n} / b_{n}=\sum_{t=0}^{m} S_{n_{m}}(t) / b_{n}+o(1)=1+o(1)
$$

in probability. Clearly also $b_{n} \sim n \nu\left(b_{n}\right)$.

The remainder of Theorem 3 we prove as follows. Since $S_{n} / b_{n} \stackrel{p}{\longrightarrow} 1$ and $n P\left(|X|>\varepsilon b_{n}\right) \rightarrow 0$ for $\varepsilon>0$, we can choose a subsequence $n_{i} \rightarrow+\infty$ for which $\sum P\left(\left|S_{n_{i}}{ }^{-b} n_{i}\right|>\varepsilon b_{n_{i}}\right)$ and $\sum n_{i} P\left(|x|>\varepsilon b_{n_{i}}\right)$ converge for every $\varepsilon>0$. Define $B(n)$ by $B(n)=b_{n_{i}}$ if $n_{i-1}<n \leq n_{i}$, so we immediately have limsup $S_{n} / B(n) \geq 1$ almost surely. Now by a version of Lévy's inequality (Lemma 2 below), since $\left(s_{n}-b_{n}\right) / b_{n} \stackrel{p}{\longrightarrow} 0$, $P\left\{\max _{i-1}^{<j \leq n_{i}}\left(s_{j}-b_{j}\right)>\varepsilon B\left(n_{j}\right)\right\} \leq P\left\{S_{n_{i}}-b_{n_{i}}>\varepsilon b_{n_{i}}\right\}+m m_{i} P\left(|x|>\varepsilon m^{-1} b_{n_{i}}\right)$.

The convergence of the series with terms on the right hand side implies the convergence of the series with terms on the left hand side, so by the Borel-Cantelli Lemma,

$$
\limsup _{i} \max _{i-1}^{<j \leq n_{i}}\left(s_{j}-b_{j}\right) / B\left(n_{i}\right) \leq 0 \text { almost surely. }
$$

Given $n>n_{1}$, choose $i=i(n)$ so that $n_{i-1}<n \leq n_{i}$. Then since $B(n)=B\left(n_{i}\right)=b_{n_{i}} \geq b_{n}$,

$$
\begin{aligned}
\underset{n}{\limsup } S_{n} / B(n) & \leq \underset{n}{\limsup }\left(S_{n}-b_{n}\right) / B\left(n_{i}\right)+\underset{n}{\limsup } b_{n} / B\left(n_{i}\right) \\
& \leq \underset{n}{\limsup } \max _{i-1}^{<j \leq n_{i}}\left(S_{j}-b_{j}\right) / B\left(n_{i}\right)+1 \leq 1 \text { almost surely, }
\end{aligned}
$$


showing that limsup $S_{n} / B(n)=1$ almost surely.

\section{Two lemmas}

LEMMA 1. For each $k$ suppose $x_{i}^{k}$ are independent random variables with finite variance $\operatorname{var} x_{i}^{k}$. Then if $m(k)$ is any sequence of integers and $s_{j}^{k}=x_{1}^{k}+x_{2}^{k}+\ldots+x_{j}^{k}$, for every real $x$, $P\left\{\max _{1 \leq j \leq m(k)}\left(S_{j}^{k}-\sum_{i=1}^{k} E X_{i}^{k}\right) \geq x\right\}$

$$
\leq 2 p\left\{S_{m(k)}^{k}-\sum_{i=1}^{m(k)} E x_{i}^{k} \geq x-\left\{2 \sum_{i=1}^{m(k)} \operatorname{var} x_{i}^{k}\right\}^{\frac{1}{2}}\right\} .
$$

Proof of Lemma 1. We omit this proof since it is similar to that of the ordinary Lévy inequality (cf. also Petrov [18]).

LEMMA 2. Let $x_{i}$ be a stationary m-dependent sequence for which $\left(S_{n}-\alpha_{n}\right) / B(n) \stackrel{p}{\longrightarrow} 0$ for constants $\alpha_{n}, B(n), B(n)>0$. Then for every $\varepsilon>0, \varepsilon<1 / 6$, there are constants $n_{0}(\varepsilon), k_{0}(\varepsilon), n_{0}>k_{0}$, for which $n \geq n_{0}$ implies for every real $x$,

(i) $\max _{k_{0} \leq k \leq n}\left|\alpha_{n}-\alpha_{k}-\alpha_{n-k}\right| \leq \varepsilon B(n)$,

$$
\text { (ii) } \begin{aligned}
(1-\varepsilon) P\left\{\max _{k_{0} \leq k \leq n}\left(s_{k}-\alpha_{k}\right) \geq x B(n)\right\} \\
\quad \leq P\left\{S_{n}-\alpha_{n} \geq(x-3 \varepsilon) B(n)\right\}+m n P\left\{\left|x_{i}\right|>\mathrm{cm}^{-1} B(n)\right\} .
\end{aligned}
$$

Proof. (i) is given in Lemma 2 of [16], and it means that $\inf _{k_{0} \leq k \leq n} P\left\{S_{n+m}-\alpha_{n}+\alpha_{k}-S_{k+m}>-\varepsilon B(n)\right\} \geq \inf _{k_{0} \leq k \leq n} P\left\{S_{n-k}-\alpha_{n-k}>-\frac{1}{2} \varepsilon B(n)\right\} \geq 1-\varepsilon$ if $n \geq n_{0}$ and $n_{0}$ is large enough, because $\left(S_{n}-\alpha_{n}\right) / B(n) \stackrel{p}{\longrightarrow} 0$. By m-dependence and stationarity, 


$$
\begin{aligned}
& (1-\varepsilon) P\left\{\max _{k_{0} \leq k \leq n}\left(s_{k}-\alpha_{k}\right)>x B(n)\right\} \\
& =(1-\varepsilon) \sum_{k=k_{0}}^{n} P\left\{S_{k}-\alpha_{k}>x B(n), \max _{k_{0} \leq j<k}\left(s_{j}-\alpha_{j}\right) \leq x B(n)\right\} \\
& \leq \sum_{k=k_{0}}^{n} P\left\{S_{k}-\alpha_{k}>x B(n), \max _{k_{0} \leq j<k}\left(s_{j}-\alpha_{j}\right) \leq x B(n), S_{n+m}-\alpha_{n}+\alpha_{k}-S_{k+m}>-\varepsilon B(n)\right\} \\
& \leq \sum_{k=k_{0}}^{n} P\left\{S_{k}-\alpha_{k}>x B(n), \max _{k_{0} \leq j<k}\left(S_{j}-\alpha_{j}\right) \leq x B(n),\right. \\
& \leq P\left\{S_{n+m}-\alpha_{n}>(x-2 \varepsilon) B(n)\right\}+n P\left\{S_{m} \leq-\varepsilon B(n)\right\} \\
& \leq P\left\{S_{n}-\alpha_{n}>(x-3 \varepsilon) B(n)\right\}+n P\left\{\left|S_{m}\right| \geq \varepsilon B(n)\right\} \\
& \leq P\left\{S_{n}-\alpha_{n}>(x-3 \varepsilon) B(n)\right\}+n m P\left(\left|x_{i}\right| \geq \varepsilon m-s_{B}+S_{k}-S_{k+m}>(x-\varepsilon) B(n)\right\} .
\end{aligned}
$$

\section{References}

[1] Y.S. Chow and Herbert Robbins, "On sums of independent random variables with infinite moments and 'fair' games", Proc. Nat. Acad. Sci. 47 (1961), 330-335.

[2] William Feller, An introduction to probability theory and its applications. Volume II, second edition (John Wiley \& Sons, New York, London, Sydney, 1971).

[3] B.V. Gnedenko, A.N. Kolmogorov, Limit distributions for sums of independent random variables, revised edition (translated by $K$. L. Chung. Addison-Wesley, Rejading, Massachusetts, London; Don Mills, Ontario; 1968).

[4] Peter Hall, "On the duality between the behaviour of sums of independent random variables and the sums of their squares", Math. Proc. Cambridge Philos. Soc. 84 (1978), 117-121.

[5] C.C. Heyde, "A note concerning behaviour of iterated logarithm type", Proc. Amer. Math. Soc. 23 (1969), 85-90. 
[6] Harry Kesten, "Sums of independent random variables - without moment conditions", Ann. Math. Statist. 43 (1972), 701-732.

[7] M.J. Klass, "Towards a universal law of the iterated logarithm, Part I", 2. Wahrsch. Verw. Gebiete 36 (1976), 165-178.

[8] M.J. Klass, "Towards a universal law of the iterated logarithm, Part II", 2. Wahrsch. Verw. Gebiete 39 (1977), 151-165.

[9] Michael Klass and Henry Teicher, "Iterated logarithm laws for asymmetric random variables barely with or without finite mean", Ann. Probab. 5 (1977), 861-874.

[10] Paul Lévy, Thêorie de l'addition des variables alèatoires (GauthierVillars, Paris, 1937).

[11] M. Loève, Probability theory I, 4th edition (Graduate Texts in Mathematics, 45. Springer-Verlag, New York, Heidelberg, New York, 1977).

[12] R.A. Maller, "A note on Karamata's generalised regular variation", $J$. Austraz. Math. Soc. Ser. A 24 (1977), 417-424.

[13] R.A. Maller, "Relative stability and the strong law of large numbers", 2. Wahrsch. Verw. Gebiete 43 (1978), 141-148.

[14] R.A. Maller, "Relative stability, characteristic functions and stochastic compactness", J. Austral. Math. Soc. Ser. A (to appear).

[15] R.A. Maller, "A note on domains of partial attraction", Ann. Probab. (to appear).

[16] R.A. Maller, "An extension of Kesten's generalised law of the iterated logarithm", Bull. Austral. Math. Soc. 21 (1980), 393-406.

[17] H.D. Miller, "A note on sums of independent random variables with infinite first moment", Ann. Math. Statist. 38 (1967), 751-758.

[18] V.V. Petrov, "A generalization of an inequality of Lévy", Theor. Prob. Appl. 20 (1975), 141-145.

[19] William E. Pruitt, "General one-sided laws of the iterated logarithm", Ann. Probab. (to appear). 
[20] B.A. Rogozin, "On the existence of exact upper sequences", Theor. Prob. App 2. 13 (1968), 667-672.

[21] B.A. Rogozin, "The distribution of the first ladder moment and height and fluctuation of a random walk", Theor. Prob. App 2. 16 (1971), 575-595.

[22] B.A. Rogozin, "Relatively stable walks", Theor. Prob. Appl. 21 (1976), 375-379.

Division of Mathematics and Statistics,

CSIRO,

Private Bag,

PO Wembley,

Western Australia 6014,

Australia. 